\title{
Asociación entre uso de cigarrillo electrónico e historia de accidente cerebrovascular en mujeres adultas de EE.UU. en el año 2018
}

\author{
Kleynner Ramón Romero[1], Sebastián Reyes Vizcaíno[1] \\ 1. Facultad de Ciencias de la Salud, Escuela de Medicina, Universidad de las Américas, Quito - Ecuador
}

Doi: https://doi.org/10.23936/pfr.v6i1.188

PRÁCTICA FAMILIAR RURAL | Vol.6 | No.1 | Marzo 2021 | Recibido: 04/02/2021 |Aprobado:18/03/2020

Cómo citar este artículo

Romero, K., Reyes, S. Asociación entre uso de cigarrillo electrónico e historia de accidente cerebrovascular en mujeres adultas de EE.UU. en el año 2018. Práctica Familiar Rural. 2021 marzo; 6(1).

Compartir en:

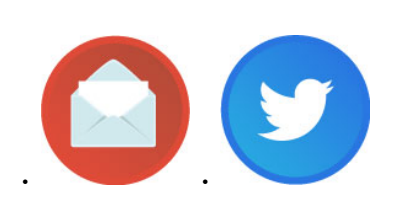

\section{Resumen}

Introducción y objetivo. El uso del cigarrillo electrónico es cada vez más común. Sólo entre los años 2010 y 2013, su uso se triplicó y pasó de 3.3\% a 8.5\%. El accidente cerebro vascular (ACV) fue en 2008 la quinta causa de muerte en Estados Unidos (EE. UU.). Sin embargo, existe escasa información sobre su relación, por lo que el objetivo del presente estudio es determinar la asociación entre el uso del cigarrillo electrónico y el desarrollo de ACV en mujeres adultas de EE. UU. que respondieron la encuesta del Behavioral Risk Factor Surveillance System (BRFSS) en el año 2018. El objetivo de este estudio fue determinar si existe una asociación entre el uso del cigarrillo electrónico y el antecedente de ACV, tomando como muestra a mujeres adultas de Estados Unidos en el 2018.

Métodos. El diseño del estudio fue un corte transversal para una muestra de 23122 mujeres mayores de 18 años en EE. UU., tomando los datos de la encuesta BFRSS del año 2018. Se 
incluyó en el estudio a las mujeres que respondieron las preguntas sobre el uso de cigarrillo electrónico (ECIGNOW) e historia de ACV (CVDSTRK3), y se excluyeron a quienes se rehusaron a responder o respondieron con la opción "Don't know / Not sure". La variable independiente fue el uso de cigarrillo electrónico, mientras que la variable dependiente fue $\mathrm{ACV}$. Las variables confusoras que se tomaron en consideración fueron edad, etnia, raza, uso de tabaco convencional o masticable, infarto de miocardio, angina y diabetes. Para este estudio se utilizaron modelos de regresión logística no ajustado y ajustado, calculando los "Odds ratio" (OR) e intervalos de confianza (IC) de 95\%.

Resultados. De 18586 mujeres que fuman cigarrillo electrónico, 894 (4.81\%) han tenido episodios de ACV, mientras que, de 4575 mujeres que no fuman cigarrillo electrónico, 208 (4.54\%) los han presentado. La asociación entre el uso de cigarrillo electrónico y ACV, después de ajustar para las variables confusoras (fumar tabaco, edad, raza, angina, infarto de miocardio y diabetes), se encontró un OR ajustado de 0.94 con un IC 95\% de 0.79-1.11. A excepción de la etnia (IC 0.83-1.67), las variables confusoras mencionadas sí mostraron asociación estadísticamente significativa con la historia de ACV.

Conclusiones. No se encontró relación estadísticamente significativa entre el uso de cigarrillo electrónico y la historia de ACV. Debido a que el estudio realizado fue de corte transversal y se tomaron datos de un solo año, se recomienda realizar un estudio de cohorte prospectiva con seguimiento a fumadores de cigarrillo electrónico, para comprobar si realmente existe o no una asociación con el desarrollo de ACV.

Palabras clave: accidente cerebrovascular; cigarrillo electrónico; nicotina

\title{
Association between e-cigarette use and history of stroke in US adult women in 2018.
}

\begin{abstract}
Introduction and objective. The use of the electronic cigarette is more and more common. Only between 2010 and 2013, its use tripled and went from $3.3 \%$ to $8.5 \%$. In 2008, cerebrovascular accident (CVA) was the fifth leading cause of death in the United States (USA). However, there is little information on their relationship, so the objective of this study is to determine the association between the use of electronic cigarettes and the development of stroke in adult women in the United States who responded to the Behavioral Risk Factor Surveillance survey. System (BRFSS) in 2018. The objective of this study was to determine if there is an association between the use of electronic cigarettes and a history of stroke, taking as a sample adult women in the United States in 2018.
\end{abstract}

Methods. The study design was a cross-sectional section for a sample of 23,122 women over 18 years of age in the US, taking data from the 2018 BFRSS survey. The women who answered the questions were included in the study. on the use of electronic cigarette (ECIGNOW) and history of stroke (CVDSTRK3), and those who refused to answer or answered with the option "Don't know / Not sure" were excluded. The independent variable was the use of electronic cigarettes, while the dependent variable was stroke. The confounding variables that were taken into consideration were age, ethnicity, race, use of conventional or chewable tobacco, myocardial infarction, angina, and diabetes. For this study, unadjusted and adjusted logistic regression models were used, calculating the "Odds ratio" (OR) and 95\% confidence intervals (CI).

Results. Of 18,586 women who smoke electronic cigarettes, 894 (4.81\%) have had episodes of stroke, while, of 4,575 women who do not smoke electronic cigarettes, $208(4.54 \%)$ have 
presented them. After adjusting for confounding variables (smoking tobacco, age, race, angina, myocardial infarction, and diabetes) the association between the use of electronic cigarettes and stroke, an adjusted OR of 0.94 was found with a $95 \%$ CI of $0.79-1.11$. With the exception of ethnicity (CI 0.83-1.67), the mentioned confounding variables did show a statistically significant association with the history of stroke.

Conclusions. No statistically significant relationship was found between the use of electronic cigarettes and the history of stroke. Because the study carried out was cross-sectional and data from a single year were collected, it is recommended to carry out a prospective cohort study with follow-up of electronic cigarette smokers, to verify whether or not there really is an association with the development of stroke.

Keywords: cerebrovascular accident; electronic cigarette; nicotine

\section{Introducción}

El cigarrillo electrónico apareció en el año 2003 en China y se ha ido popularizando de manera exponencial gracias a su atractivo diseño y variedad de sabores, por lo que ha logrado insertarse tanto en poblaciones jóvenes, como en aquellos grupos que nunca habían probado tabaco convencional [1]. El uso del cigarrillo electrónico es cada vez más común, estimando 10.8 millones de usuarios en Estados Unidos de América (EE. UU.) en el 2016 [2]. La justificación de algunos consumidores para este incremento, además de la popularización del producto, es que el cigarrillo electrónico los ayuda con el cese del tabaquismo convencional como lo demuestra un estudio con 2501 participantes, publicado en el 2015 por la Sociedad de investigación en nicotina y tabaco [3]. Sin embargo, a pesar de que el cigarrillo electrónico se considera una alternativa más saludable al tabaco, se sabe poco sobre sus efectos sobre la salud a corto y largo plazo [4].

Entre las consecuencias que conlleva el uso del tabaco combustible, una de las más devastadoras para la salud es el ACV, que se define como el daño de una o varias zonas cerebrales por la falta de irrigación sanguínea, ya sea por hemorragia o embolismo [5]. Según los artículos "Nicotine and electronic cigarette (ECig) exposure decreases brain glucose utilization in ischemic stroke" publicado en 2018 en la revista "Journal of Neurochemistry" y "Stroke Epidemiology and Risk Factor Management", publicado por la Academia Americana de Neurología en el 2017, el ACV es la quinta causa de muerte en EE. UU. [4][6]. Cabe mencionar que el cigarrillo electrónico no quema tabaco mientras se lo consume, dado que tiene un líquido llamado "esencia de vapeo" que es calentado gracias a su batería recargable hasta el punto de ser vaporizado [1]. Sin embargo, al igual que en el caso del tabaco, el componente principal del cigarrillo electrónico es la nicotina (además de propilenglicol, glicerol, etilenglicol y saborizantes), por lo que cabe la sospecha de que el ACV también pudiera ser consecuencia del uso de cigarrillo electrónico [4][7][8].

La nicotina llega al cerebro atravesando la barrera hematoencefálica y causando una cascada de reacciones bioquímicas que predisponen al paciente a un accidente cerebrovascular [7]. Estudios preclínicos han demostrado que la nicotina contenida tanto en el tabaco, como en los cigarrillos electrónicos puede predisponer al desarrollo de especies reactivas de oxígeno (ROS) en el cerebro, lo cual puede conllevar a un estrés oxidativo que favorezca el desarrollo de ACV [7]. Esta condición, se sospecha, empeora con la diabetes ya que se enlentece el metabolismo cerebral de glucosa al disminuir la glucólisis, la expresión de los transportadores de glucosa GLUT1 neuronal y la expresión de $\alpha 7 \mathrm{nAChR}$ regulada [4]. Por esta razón se considera especialmente riesgoso el consumo de nicotina para los pacientes que sufren de diabetes mellitus.

La literatura actual se mantiene indeterminada en cuanto al impacto de los cigarrillos electrónicos para el desarrollo de ACV, sin embargo, existen algunos estudios básicos al respecto como reportes de casos aislados y resúmenes científicos. Se encontró un resumen científico donde utilizaron los datos de la encuesta 
BRFSS del CDC del año 2016 para evaluar esta relación [9]. En este estudio se encontró relación entre el uso del cigarrillo electrónico y el desarrollo de ACV \{Odds Ratio (OR) 1.71 [Intervalo de confianza (IC) $1.64-1.8$ ] [9]. Por otro lado, en un estudio comparativo entre fumadores de tabaco, usuarios de cigarrillo electrónico y no fumadores, los resultados arrojaron una mayor probabilidad de desarrollar ACV entre los fumadores de tabaco, que en los usuarios de cigarrillo electrónico; no obstante, en comparación con no fumadores y con fumadores de cigarrillo combustible, se demostró un mayor riesgo estadísticamente significativo de desarrollar ACV entre los usuarios de cigarrillo electrónico y tabaco combustible [8].

El objetivo principal del presente estudio fue determinar si existe una asociación entre el uso del cigarrillo electrónico y la historia de ACV, tomando en cuenta distintas variables confusoras que pudieran sesgar los resultados, tales como fumar tabaco, edad, raza, angina, infarto de miocardio y diabetes. La hipótesis nula de este estudio fue que no existe asociación entre el consumo de cigarrillo electrónico y la historia de ACV, mientras que la hipótesis alternativa es que el consumo de cigarrillo electrónico tiene relación con la historia de ACV. A pesar de que el desarrollo de ACV es más frecuente en hombres que en mujeres en edades tempranas, son ellas quienes tienen un mayor riesgo de sufrir esta afección a lo largo de la vida [6]. Es por esta razón que este estudio está centrado en personas de sexo femenino. Por lo antes mencionado, es importante seguir desarrollando investigación científica y bioestadística en cuanto al tema, dado que el cigarrillo electrónico es un dispositivo relativamente nuevo y no se cuenta con evidencia suficiente sobre sus riesgos.

\section{Métodos}

Se realizó un estudio observacional de corte transversal utilizando datos de la encuesta BRFSS publicada por la CDC en EE. UU. en el año 2018 [10]. Para este estudio se incluyeron solo mujeres que respondieron a las preguntas: ECIGNOW ("Do you now use e-cigarettes, every day, some days, or not at all?") y CVDSTRK3 ("Ever Diagnosed with a Stroke") y se excluyeron a las que eligieron la opción "Don't know / Not sure". Para el estudio se tomaron 23122 mujeres que fueron quienes cumplieron con los criterios de inclusión y exclusión, ya que 23225 respondieron a la pregunta del cigarrillo electrónico y 238209 mujeres respondieron a la pregunta de $\mathrm{ACV}$, sin embargo, de ellas, solamente 23122 respondieron adecuadamente ambas preguntas. En cuanto a las variables confusoras, se compararon tanto con la variable independiente, como con la dependiente, por lo que el número de participantes que se eligió para cada una fue el número de mujeres que respondieron la pregunta correspondiente a la variable confusora y la correspondiente a la variable con la que se estaba comparando (tanto dependiente como independiente).

Nuestra variable independiente fue el consumo de cigarrillo electrónico (ECIGNOW), mientras que nuestra variable dependiente fue la historia de ACV (CVDSTRK3). Para este estudio, las variables confusoras fueron: el consumo de tabaco convencional (SMOKDAY2: "Frequency of Days Now Smoking"), edad (AGE_G: "Imputed age in six groups"), etnia (_HISPANC: "Hispanic, Latino/a, or Spanish origin calculated variable"), historia de infarto de miocardio (CVDINFR4: "Ever Diagnosed with Heart Attack"), angina $\mathrm{u}$ otra enfermedad coronaria (CVDCRHD4: "Ever Diagnosed with Angina or Coronary Heart Disease") y la historia de diabetes (DIABETE3: "(Ever told) you have diabetes"). Se dicotomizaron todas las preguntas cualitativas (ECIGNOW, SMOKDAY2, AGE_G), para facilitar el análisis estadístico. Las variables ECIGNOW y SMOKDAY2 se dicotomizaron en "uso" y "no uso", considerando a quienes escogieron la opción "some days" como usuarios. Por su parte, la variable AGE G se dicotomizó en menores de "55 años" y "55 años o más", pues sabemos que la edad avanzada es un factor de riesgo para el desarrollo de ACV.

Se evaluó únicamente a partir de las respuestas que los participantes proveyeron a la encuesta del BRFSS del 2018 en EE. UU. y el análisis estadístico se realizó mediante el programa RStudio para obtener OR, intervalos de confianza y el valor p para esta relación. 
La revisión de literatura fue realizada mediante PubMed y Google Scholar, utilizando las palabras clave "stroke", "nicotine" y "electronic cigarette". Esto arrojó escasa información acerca de estos temas en conjunto, pues la búsqueda en PubMed no desplegó resultados utilizando los términos MeSH con operadores booleanos: "stroke AND electronic cigarrette AND nicotine" o "stroke AND electronic cigarrette"; y solamente desplegó 27 resultados usando los términos y operadores booleanos: "stroke AND electronic cigarrette OR nicotine", pero la mayoría trataba sobre el ACV y la nicotina del cigarrillo convencional. De igual manera en Google Scholar se encontró poca literatura que trataba en específico sobre el cigarrillo electrónico y su relación con ACV, pero se encontraron varios estudios sobre los temas por separado. De todos los artículos encontrados que trataban estos temas en PubMed y Google Scholar, tanto en conjunto como por separado, se escogieron los que mejor se adaptaron a nuestro estudio.

\section{Resultados}

En total, 437436 personas respondieron a la encuesta BRFSS del 2018, de las cuales 238911 fueron mujeres. Del total de mujeres que respondieron a la encuesta, 23225 respondieron a la pregunta del cigarrillo electrónico (ECIGNOW) con las opciones "every day", "some days" o "not at all" y 238209 mujeres a la pregunta de ACV (CVDSTRK3) de forma afirmativa o negativa. De ellas, solamente 23122 respondieron ambas preguntas de acuerdo con nuestros criterios de inclusión y exclusión antes mencionados, por lo que la muestra final incluyó ese número de participantes.

Tabla 1. El consumo de cigarrillo electrónico se observa más en fumadores de tabaco $(65,54 \%$ vs $34,46 \%$ $[p<0,001])$, en menores de 55 años $(85,05 \%$ vs $14,95 \%$ [p<0,001]) y en no diabéticos $(86,92 \%$ vs $13,08 \%$ $[\mathrm{p}=0,003])$, mientras que la etnia, historia de angina y la historia de infarto mostraron un valor $\mathrm{p}$ mayor a 0,05 por lo que su relación con el consumo de cigarrillo electrónico no se considera estadísticamente significativa.

Tabla 1. Asociación entre posibles confusores y el uso de cigarrilllo ellectrónico.

\section{Cigarrillo electrónico}

\begin{tabular}{|c|c|c|c|c|}
\hline Características & $\begin{array}{l}\text { Uso }(\%) \\
(n=18635)\end{array}$ & $\begin{array}{l}\text { No uso }(\%) \\
(n=4590)\end{array}$ & $\begin{array}{l}\text { TOTAL } \\
(\mathrm{n}=23225)\end{array}$ & Valor $\mathrm{p}$ \\
\hline \multicolumn{5}{|c|}{ Cigarrillo combustible } \\
\hline Uso & $9391(66,46 \%)$ & $2368(62,12 \%)$ & 11759 & \\
\hline No uso & $4739(25,12 \%)$ & $1444(27,47 \%)$ & 6183 & $0,001^{*}$ \\
\hline Total & $14130(100 \%)$ & $3812(100 \%)$ & 17942 & \\
\hline \multicolumn{5}{|c|}{ Edad (años) } \\
\hline 18-54 & $3101(68,15 \%)$ & $12021(64,51 \%)$ & 19742 & \multirow{4}{*}{$0,001 *$} \\
\hline 55 o mayor & $1449(31,85 \%)$ & $6614(35,49 \%)$ & 3469 & \\
\hline Total & $18621(100 \%)$ & $4590(100 \%)$ & 23211 & \\
\hline & & Etnia & & \\
\hline Hispano & $1274(6,89 \%)$ & $284(6,21 \%)$ & 1558 & \multirow{3}{*}{0,112} \\
\hline No hispano & $17227(48,22 \%)$ & $4287(48,40 \%)$ & 21514 & \\
\hline Total & $18501(100 \%)$ & $4571(100 \%)$ & 23072 & \\
\hline
\end{tabular}




$\begin{array}{lllll}\text { Si } & 901(4,85 \%) & 206(4,52 \%) & 1107 & 0,338 \\ \text { No } & 17678(95,15 \%) & 4373(95,48 \%) & 22051 & \\ \text { Total } & 18579(100 \%) & 4579(100 \%) & 23158 & \\ & & \text { Angina } & & \\ \text { Si } & 817(4,41 \%) & 203(4,47 \%) & 1020 & \\ \text { No } & 17695(95,59 \%) & 4350(95,53 \%) & 22045 & \\ \text { Total } & 18512(100 \%) & 4553(100 \%) & 23065 & 0,003 \\ & & \text { Diabetes } & & \\ \text { Si } & 527(11,61 \%) & 2496(13,42 \%) & 3032 & \\ \text { No } & 4012(88,39 \%) & 16105(86,58 \%) & 20148 & \\ \text { Total } & 4579(100 \%) & 18601(100 \%) & 23180 & \end{array}$

*Los valores de $\mathrm{p}$ menores a 0,001 se representan de esta manera

Tabla 2. Tras el análisis estadístico para determinar los OR e IC de la relación entre el consumo de cigarrillo combustible e historia de ACV, no se encontró asociación estadísticamente significativa entre las variables dependiente e independiente (0.93 [0.80-1.10]), incluso luego de haber ajustado para las variables confusoras antes mencionadas (OR 0.94 [0.79-1.11])

\section{Tabla 2. Asociación ajustada y no ajustada entre cigarrilllo electrónico y ACV}

$\begin{array}{lccc}\text { Características } & \text { No ajustado } & \text { Ajustado1 } & \text { Valor P } \\ \text { Cigarrillo electrónico } & \text { OR (95\% IC) } & \text { OR (95\% IC) } & \\ \text { No Uso } & \text { REF2 } & \text { REF2 } & 0,466 \\ \text { Uso } & 0,93(0,80-1.10) & 0,94(0,79-1,11) & \end{array}$

1. Los valores se ajustaron para todas las variables confusoras presentadas en la tabla 4.

2. Valores de referencia

Tabla 3. Las variables confusoras demostraron, en su mayoría, relación con ACV, a excepción de la etnia que no mostró relación estadísticamente significativa (OR 1,18 [0.83-1.67]); por su parte, la historia de infarto (OR 3,31 [2.70-4.06]) y de angina (OR 2,32 [1.87-2.88]) fueron las variables más relacionadas con ACV, mientras que, la edad (OR 1,99 [1.71-2.31)], la diabetes (OR 1,91 [1.64-2.23]) y el consumo de tabaco combustible (OR 1,18 [1.02-1.37]) demostraron asociación con ACV, pero en menor proporción.

\section{Tabla 3. Asociación ajustadla entre variables confusoras y ACV}

\section{Características $\quad$ Ajustado1 OR (95\% IC)}

Cigarrillo combustible

$$
\text { Uso } 1,18(1,02-1,37)
$$

No uso

REF2

Edad (años) 
18-54

55 o mayor

Hispano

No hispano

Sí

No

Sí

No

Sí

No
REF2

$1,99(1,71-2,31)$

Etnia

$1,18(0,83-1,67)$

REF2

IM

$3,31(2,70-4,06)$

REF2

\section{Angina}

$2,32(1,87-2,88)$

REF2

\section{Diabetes}

$1,91(1,64-2,23)$

REF2

1. Los valores se ajustaron para todas las variables confusoras presentadas en esta tabla.

2 Valores de referencia

\section{Discusión}

A diferencia del estudio realizado en enero del 2019 para el journal de la "American Heart Association" (AHA) "Electronic Cigarette Use is Associated with a Higher Risk of Stroke" en el que se encontró que los usuarios de cigarrillo electrónico presentaron una probabilidad mayor de historia de ACV (OR 1.71 [1.641.8]) ajustando para las variables edad, sexo, consumo de tabaco, diabetes, práctica de ejercicios e índice de masa corporal (IMC) [9], este estudio no encontró relación estadísticamente significativa entre el consumo de cigarrillo electrónico y ACV. Sin embargo, en el estudio comparativo "Risk of Stroke With E-Cigarette and Combustible Cigarette Use in Young Adults" publicado en la "American Journal of Preventive Medicine" en el 2019, tampoco se encontró relación estadísticamente significativa entre el uso de cigarrillo electrónico y ACV (OR 0.69 [0.34-1.42]) [8]. Esto indica que la literatura es controversial en cuanto a esta relación, pues existen diversos factores que influyen a la aparición de esta enfermedad, los cuales no han podido ser tomados en cuenta en los estudios realizados anteriormente [6]. En cuanto al presente estudio, la relación no se pudo estimar de una manera significativa debido a que no se pudieron tomar en cuenta distintos factores fundamentales como son: la frecuencia con la que los participantes utilizan el cigarrillo electrónico, la edad de inicio del consumo, la cantidad que cada uno consume, ni el tiempo en que lo llevan consumiendo, pues se trató de un estudio de corte transversal.

En el mercado existen cigarrillos electrónicos que contienen nicotina en diferentes concentraciones [1]. Sin embargo, a pesar de no contener tabaco en sí y no consumirse por medio de combustión, los cigarrillos electrónicos contienen nicotina, sustancia que se sabe que provoca daño causal del endotelio vascular en dependencia de la dosis, debido a inflamación y acumulación de ROS en este tejido [7]. Esto favorece el desarrollo de afecciones vasculares y patologías cardíacas como angina, infarto de miocardio y formación de trombos [2][7], factores que mostraron una gran asociación con el desarrollo de ACV y posterior daño cerebral en otros estudios mencionados [2][4][6][7]. 
Las variables confusoras tomadas para este estudio para ajustar los resultados principales y tener resultados más fiables, demostraron en su mayoría relación con la historia de ACV, lo cual era esperado debido a la gran evidencia que se encontró sobre esta relación, pues varios artículos mencionaban el uso de tabaco, la edad, la diabetes, las afecciones cardiacas como angina o infarto de miocardio como factores de riesgo para el desarrollo de ACV [2][4][5][6][7][8][9]. Dado que se sabe que la raza es determinante en cuanto al riesgo de ACV, como se indica en el artículo "Stroke Epidemiology and Risk Factor Management" [6], se hizo el ajuste también para la variable de etnia, pues ésta se encontraba dicotomizada en la encuesta BRFSS en "hispanos" y "no hispanos" [10]. Sin embargo, en el presente estudio esta variable no demostró ser un factor estadísticamente determinante en relación con la historia de ACV, lo que podría significar que esta asociación está dada por la raza negra específicamente y no por la etnia [6]. Por otra parte, solo la mitad de las variables confusoras demostraron relación estadísticamente significativa con el consumo de cigarrillo electrónico (consumo de tabaco, edad y diabetes), pues es sabido que éste se da en su mayoría en fumadores previos de tabaco, así como en personas jóvenes [1][3]. No obstante, la relación encontrada entre el uso de cigarrillo electrónico y la historia de diabetes fue un resultado que no se había anticipado, pues el hecho de tener diabetes no pareciera relacionarse con un menor consumo de cigarrillo electrónico, pero sí podría deberse a que en general los pacientes diabéticos están en el grupo de los mayores de 55 años, donde el consumo de cigarrillo electrónico no es muy popular por ser un producto relativamente nuevo [1][3].

Dado que nuestro estudio se adaptó a la encuesta BRFSS realizada por la CDC en el año 2018, solo se pudieron tomar variables confusoras que pudieran tener concordancia con las preguntas ya establecidas, lo cual representa una limitación grande para el estudio, pues no se pudo tomar en cuenta un factor de riesgo fundamental para el desarrollo de ACV como la hipertensión arterial (HTA), pues no había pregunta en la encuesta que se relacionara con esa afección [6]. Por otro lado, no se tomó en cuenta otras variables que influyen para el desarrollo de ACV como la condición física y el IMC, pues son variables muy poco confiables, al ser una encuesta telefónica [6]. Cabe destacar también que se desconoce la veracidad de las respuestas proporcionadas por las participantes, a pesar de que la encuesta BRFSS es anónima, lo cual minimiza esta posibilidad. Además, el número de personas que sufrió ACV es muy pequeña en esta muestra, lo cual pudo influir para que el resultado no haya demostrado una asociación estadísticamente significativa entre las dos variables. A pesar de esto, el actual estudio es importante debido a que presenta evidencia estadística en cuanto a la correlación entre el consumo de cigarrillo electrónico y la historia de ACV, y se la puede comparar con la correlación estadística que demuestran otras variables que han sido señaladas en distintos estudios como factores que aumentan el riesgo de ACV, tales como el consumo de tabaco, la edad, historia de diabetes o de patologías cardíacas [2][4][5][6][7][8][9].

Con los resultados obtenidos en este estudio no podemos rechazar nuestra hipótesis nula, pues no se encontró asociación estadísticamente significativa entre el uso de cigarrillo electrónico y la historia de ACV, por lo que recomendamos realizar estudios de cohorte prospectiva con seguimiento a fumadores de cigarrillo electrónico, tomando en cuenta factores de riesgo como la edad, el consumo de tabaco, obesidad, apnea de sueño, sedentarismo, diabetes, coagulopatías, dislipidemias, malnutrición, antecedentes de hipertensión arterial y otras patologías cardiovasculares para comprobar si realmente existe o no una asociación entre el uso de este artefacto y el desarrollo de ACV [2][6].

\section{Aspectos éticos}

El presente trabajo fue realizado bajo los cuatro criterios bioéticos de autonomía, no maleficencia, beneficencia y justicia. No existe ningún riesgo en el estudio, pues no existe ningún tipo de intervención por parte de los participantes ni de los investigadores. Se trata de una revisión de datos obtenidos previamente de las encuestas de la BRFSS del 2018, los cuales permanecen siempre anónimos. Declaramos que no tenemos ningún conflicto de interés al realizar esta investigación.

\section{Referencias bibliográficas}


1. Rigotti, N., Kalkhoran, S., Vaping and e-cigarettes. Tomado de UpToDate el 22 de junio de 2020 de: https://www.uptodate.com/contents/vaping-and-e-cigarettes?

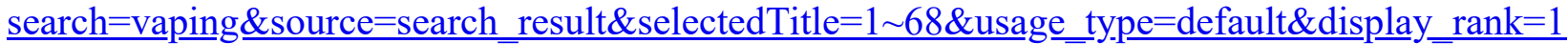

2. Osei A, Mirbolouk M, Orimoloye O, et al. Association Between E-Cigarette Use and Cardiovascular Disease Among Never and Current Combustible-Cigarette Smokers. The American Journal of Medicine. 2019;132(8):949-954.e2.

3. Berg, C., Haardoerfer, R., et al. Cigarette Users' Interest in Using or Switching to Electronic Nicotine Delivery Systems for Smokeless Tobacco for Harm Reduction, Cessation, or Novelty: A CrossSectional Survey of US Adults. Oxford University Press on behalf of the Society for Research on Nicotine and Tobacco 2015;17:245-255

4. Sifat A, Vaidya B, Kaisar M, Cucullo L, Abbruscato T. Nicotine and electronic cigarette (E-Cig) exposure decreases brain glucose utilization in ischemic stroke. Journal of Neurochemistry. 2018;147(2):204-221.

5. Cook K, Bhatti L, Tursan d'Espaignet E. Resúmenes informativos de la OMS sobre el tabaco: tabaco y accidentes cerebrovasculares. OMS, Ginebra, 2016

6. Guzik A, Bushnell C. Stroke Epidemiology and Risk Factor Management. CONTINUUM: Lifelong Learning in Neurology. 2017;23(1):15-39.

7. Kaisar M, Villalba H, Prasad S, et al. Offsetting the impact of smoking and e-cigarette vaping on the cerebrovascular system and stroke injury: Is Metformin a viable countermeasure? Redox Biology. 2017; 13:353-362.

8. Parekh T, Pemmasani S, Desai R. Risk of Stroke With E-Cigarette and Combustible Cigarette Use in Young Adults. American Journal of Preventive Medicine. 2019;58(3):446-452.

9. Ndunda P, Muutu T. Abstract 9: Electronic Cigarette Use is Associated with a Higher Risk of Stroke. Stroke. 2019;50 (In press).

10. Centers for Disease Control and Prevention (CDC). Behavioral Risk Factor Surveillance System Survey Data. Atlanta, Georgia: U.S. Department of Health and Human Services, Centers for Disease Control and Prevention, 2018.

\section{Abreviaciones}

\section{Término}

Accidente cerebrovascular

American Heart Association

Behavioral Risk Factor Surveillance System

Glucotransportadores

Hipertensión arterial

Infarto Agudo de Miocardio

Índice de masa corporal

Medical Subject Headings

Especies reactivas de oxígeno

Estados Unidos de América

\section{Abreviación}

$\mathrm{ACV}$

AHA

BRFSS

GLUT

HTA

IAM

IMC

$\mathrm{MeSH}$

ROS

EE. UU.

\section{Anexos}

Anexo 1. De las 23122 participantes, el 0.88\% (204) afirmó haber sufrido un ACV y haber usado cigarrillo electrónico, mientras que el 3,87\% (894) afirmó haber sufrido un ACV, pero negó utilizar cigarrillo 
electrónico. Por otro lado, el 95,25\% (22024) de las participantes no había sufrido ACV, a pesar de que el 19,67\% (4332) de ellas consumía cigarrillo electrónico.

Anexo 1. Asociación entre uso de cigarrilllo electrónico y posibles confusores, con historia de ACV.

\section{Características}

No uso

Uso

Total

Uso

No uso

Total

18-54

55 o mayor

Total

Hispano

No hispano

Total

Sí

No

Total

Sí

No

Total

Sí

No

Total
Sí ACV (\%)

No ACV (\%)

Total

Valor $\mathrm{p}$

\section{Cigarrillo Electrónico}

$894(81,42 \%)$
$204(18,58 \%)$
$1098(100 \%)$

$17692(80,33 \%)$

18586

4536

0,396

23122

\section{Combustible}

$1973(38,27 \%)$

$28623(34,68 \%)$

30596

$3182(61,73 \%)$

$53919(65,32 \%)$

57101

$0,001^{*}$

$5155(100 \%)$

$82542(100 \%)$

87697

\section{Edad (años)}

$1719(16,52 \%)$

$97578(42,83 \%)$

99297

$8686(83,48 \%)$

$130226(57,17 \%)$

138912

$0,001^{*}$

$10405(100 \%)$

$225898(100 \%)$

238209

\section{Etnia}

$485(4,71 \%)$

$19215(8,51 \%)$

19700

$9816(95,29 \%)$

$206683(91,49 \%)$

216499

$0,001 *$

$10301(100 \%)$

$225898(100 \%)$

236199

Infarto de miocardio

$2261(22,03 \%)$

$8283(3,65 \%)$

10544

$8003(77,97 \%)$

$218805(96,35 \%)$

226808

$0,001 *$

$10264(100 \%)$

$227088(100 \%)$

237352

\section{Angina}

$2118(20,90 \%)$

$9165(4,05 \%)$

11283

$8014(79,10 \%)$

$217202(95,95 \%)$

225216

$0,001^{*}$

$10132(100 \%)$

$226367(100 \%)$

236499

\section{Diabetes}

$3373(32,47 \%)$

32327 (14,21\%)

35700

$7015(67,53 \%)$

$195228(85,79 \%)$

202243

$0,001^{*}$

$227555(100 \%)$

237943

*Los valores de p menores a 0,001 se representan de esta manera. 